\title{
Can Urodynamic Findings of Overactive Bladder Determine the Treatment Effect of Urotherapy in Children?
}

\author{
Chun Wei1,2,3,4, Jie Gao1,2,3,4, Lihua Kou1,2,3,4, Xing Liu1,2,3,4, Tao Lin1,2,3,4 and Dawei He1,2,3,4 \\ 1Department of Pediatric Urology, Children's Hospital of Chongqing Medical University, China \\ ${ }^{2}$ Ministry of Education Key Laboratory of Child Development and Disorders, Chongqing, 400014 China \\ ${ }^{3}$ Chongqing Key Laboratory of Children Urogenital Development and Tissue Engineering, China \\ ${ }^{4}$ China International Science and Technology Cooperation Base of Child Development and Critical Disorders, China
}

\begin{abstract}
Objective: To assess the correlation between urodynamic study findings and urotherapy treatment response in children with urotherapy-resistant overactive bladder $(\mathrm{OAB})$.

Study Design: A cross-sectional study.

Place and Duration of Study: Department of Pediatric Urology, Children's Hospital of Chongqing Medical University, Chongqing, China, from January 2016 to March 2018.

Methodology: The study included 232 children presented with urotherapy-resistant OAB defined as (completing threemonth standard urotherapy with sustained $O A B$ symptoms. After excluding urinary infection and neurogenic, anatomical abnormalities, urodynamic study (UDS) was proceeded to record DO (detrusor overactivity) and maximum CBC (cystometry bladder capacity), pelvic floor awareness was guided during UDS. Following UDS, standard urotherapy and pelvic floor training was continued for another month and treatment effect was evaluated.

Results: At the end of one-month; of urotherapy following UDS, significant response (SR) was observed in $57.3 \%$ (133/232) children. As $28.9 \%$ (67/232) children showed partial response (PR), the other $13.8 \%$ (32/232) children showed no response (NR). The results showed that patients with normal bladder function or DO respond equally well to urotherapy. The percentage of significant response (SR) was $68.9 \%(93 / 135)$ and $60.0 \%(27 / 45)(p=0.148)$, which was significantly better than children with decreased CBC (DCBC) (SR: 33.3\%, 10/30) or DO+DCBC (SR: 13.6\%, 3/22, $p=0.000)$.

Conclusion: Urodynamic verification of DO is not a prerequisite for urotherapy treatment response. Decreased cystometry bladder capacity (DCBC) found in UDS may suggest a poorer treatment response to urotherapy than patients with normal CBC.
\end{abstract}

Key Words: Overactive bladder, Urodynamic, Overactive detrusor, Decreased cystometry bladder capacity, Urotherapy.

How to cite this article: Wei C, Gao J, Kou L, Liu X, Lin T, He D. Can urodynamic findings of overactive bladder determine the treatment effect of urotherapy in children? J Coll Physicians Surg Pak 2020; 30(1):41-45.

\section{INTRODUCTION}

Overactive bladder $(\mathrm{OAB})$ is a common medical condition characterised by urinary urgency, usually accompanied by frequency and nocturia, with or without urinary incontinence, in the absence of urinary tract infection (UTI) or other obvious pathology.1,2 OAB appears to have a peak incidence that occurs between 5 and 7 years of age, ${ }^{3}$ which can have influence on physical and mental health. ${ }^{4} \mathrm{~A}$ child with $\mathrm{OAB}$ has a very good chance of becoming an adult who continues to have the problem of OAB. 5

Though mostly regarded as a self-limiting condition that resolves in most cases over time, the symptom could last for months to years, which can disrupt daily activities

Correspondence to: Jie Gao, Department of Pediatric Urology, Children's Hospital of Chongqing Medical University,

Chongqing, 400014, China

E-mail:1909747329@qq.com

Received: April 03, 2019; Revised: October 25, 2019;

Accepted: December 04, 2019 and decrease the quality of life. ${ }^{6}$ Urotherapy, biofeedback and neuromodulation are frequently used non-pharmacological treatment strategies in clinical practice. Urotherapy is still the first line treatment choice for carrying little risk of adverse risks and the potential benefits. ${ }^{7}$ Bladder training and pelvic floor muscle training have also showed significant treatment response. 8

As resistant $O A B$ may bring confusion to clinical treatment, 9 further understanding of lower urinary tract function may be instructive for treatment and provide more professional support, which will increase patients' persistence and adherence to other treatment. ${ }^{10}$

The aim of the study was to determine if urodynamic study findings could influence the treatment effect in urotherapy-resistant overactive bladder.

\section{METHODOLOGY}

It was a cross-sectional study conducted from January 2016 to March 2018 at the Department of Pediatric Urology, Children's Hospital of Chongqing Medical 
University, Chongqing, China. The study was approved by the Ethics Committee of Hospital. Informed consents were given by all patients' parents. All patients were diagnosed with $O A B$ by symptoms, according to the ICCS guidelines. A total of 232 patients with an average age of 76 months (36-168 months), referring with complaints of urotherapy-resistant $O A B$, were enrolled into this study. The inclusion criteria were toilet-trained children with $O A B$ symptoms resistant to three to fivemonth standard urothearpy, The exclusion criteria were children with enuresis and constipation with neurological disease, possible neuropathy, urinary infection or anatomic abnormality of the urinary tract.

When first diagnosed with $O A B$, patients were educated about normal LUT function and how they deviated from normal. Then they were treated with standard urotherapy which comprised education of the child and family, appropriate fluid intake, regular optimal voiding regimens and bowel programmes for three to five months. No other specific interventions of urotherapy such as pelvic floor muscle awareness, biofeedback training, and neuromodulation were used.

After three- to five-month standard urothearpy, if symptoms of OAB persisted, then UDS was carried out. With patient laying 30 degrees supine, a 6-7 Fr doublelumen catheter was inserted into the bladder to record bladder pressure, and a small (8 Fr) rectal balloon catheter was placed to record the abdominal pressure and pelvic floor pressure. Temperature of the infusate was about $27^{\circ} \mathrm{C}$ to $30^{\circ} \mathrm{C}$. The filling rate was $5 \%$ of expected bladder capacity (EBC) per minute, the standard formula for calculating expected bladder capacity is $\mathrm{EBC}=$ age (years) $\times 30+30$ (expressed in $\mathrm{ml}$ ). During infusion, to reduce anxiety, patients were accompanied by parents and provided with some videos. DO and CBC (cystometry bladder capacity) were recorded during cystometry, $\mathrm{CBC}$ was defined as the volume of urine drained after the UDS and was compared with the maximum voiding volume (MVV) recorded by parents. If $\mathrm{CBC}$ was markedly less than voiding volume and the patient was anxious, then catheters were kept and patients were relaxed to adapt to the UDS environment. The second UDS was conducted about 30 minutes later, and the infusion rate was about half rate of the first time to prolong the infusion. The maximum cystometric capacity was recorded as $\mathrm{CBC}$. When $\mathrm{CBC} / \mathrm{EBC} \leq 60 \%$, it was defined as DCBC (decreased cystometry bladder capacity). DO was defined as the occurrence of involuntary detrusor contractions during cystometry. Children without DO or DCBC were defined as having normal bladder function.

During UDS, when patients felt urgency or urgency signs such as adduction of toes or clamping perineum were observed, then infusion was paused, and children were guided to contract the pelvic floor muscle. An increase of the pressure recorded by the rectal balloon catheter suggested that the pelvic floor contraction was effective. Infusion was restarted when urgency disappeared. After the urodynamic evaluation, children were treated with standard urotherapy and specific intervention (contract pelvic floor muscle when urgency felt) for another month. All patients were evaluated by clinical symptoms just after the one-month standard urotherapy and pelvic floor training following the UDS. When evaluating the results of treatment response, significant response (SR) was defined as reduction of the complaints more than $90 \%$. The result was regarded as partial response (PR) when complaints reduced by $50 \%$ to $90 \%$. No response (NR) was defined as reduction of complaints less than $50 \%$.

Statistical descriptions of enumeration data were expressed by proportion. Pearson Chi-Square (for categorical data), Kruskal-Wallis and Mann-Whitney (for continuous data) were used for statistical analysis through the software package of SPSS 19.0. Spearman's correlation was run to analyse the relationship between the patient's age and treatment response. The level of significance was set at 0.05 .

\section{RESULTS}

During the time period, 965 children were seen in our centre for complaints of urgency, but only 232 children were included into this study. Of the 232 children (158 boys, 74 girls), the ratio of overactive bladder in boys and girls is $2.14: 1$. The age distribution was $37.1 \%(86 /$ 232) children ranging from 3 to 5 years, $35.3 \%(82 / 232)$ children from 5 to 7 years, with the leftover $27.6 \%$ (64/232) children older than 7 years. The prevalence of OAB decreased in older children.

Of the 232 children, urodynamic studies of the lower urinary tract revealed bladder dysfunction in $41.8 \%$ (97/232) children, of which $19.4 \%$ (45/232) children were found with DO, $12.9 \%(30 / 232)$ children with DCBC, and the leftover $9.5 \%(22 / 232)$ children with DCBC as well as DO. No statistical differences were observed between boys and girls (Table I).

The urodynamic findings of OAB for different age groups were as follows: For the 86 children ranging from 3 to 5 years, $60.5 \%(52 / 86)$ were normal, $25.6 \%(22 / 86)$ were found with $\mathrm{DO}$, and $13.9 \%$ (12/86) were found with $\mathrm{DCBC}$ or DO+DCBC. For the 82 children ranging from 5 to 7 years, $58.5 \%(48 / 82)$ were normal, $19.5 \%(16 / 82)$ with DO, and the leftover $21.9 \%(18 / 82)$ with DCBC or DO+DCBC. For the 64 children older than 7 years, $54.7 \%$ (35/64) were normal, $10.9 \%$ (7/64) with DO, but the rate of DCBC or DO+DCBC was up to $34.4 \%$ (22/64). Pearson Chi-square test was used to compare the ratio of different urodynamic findings for various age groups, no statistically significant difference of normal bladder function or DO was observed between different age groups $(p=0.775,0.081)$. But for children older than 
Table I: Urodynamic study findings of the 232 children with overactive bladder.

\begin{tabular}{l|c|c|c|c|c}
\hline Gender $(\mathrm{n})$ & $\begin{array}{c}\text { Normal } \\
\mathrm{n}(\%)\end{array}$ & $\begin{array}{c}\text { DO } \\
\mathrm{n}(\%)\end{array}$ & $\begin{array}{c}\text { DCBC } \\
\mathrm{n}(\%)\end{array}$ & $\begin{array}{c}\text { DO + DCBC } \\
\mathrm{n}(\%)\end{array}$ & $\mathrm{p}$-value \\
\hline Male (158) & $94(59.5)$ & $30(19.0)$ & $21(13.3)$ & $13(8.2)$ & $0.787 \#$ \\
Female (74) & $41(55.4)$ & $15(20.2)$ & $9(12.2)$ & $9(12.2)$ & \\
Total (232) & $135(58.2)$ & $45(19.4)$ & $30(12.9)$ & $22(9.5)$ & \\
\hline
\end{tabular}

\# Pearson Chi-square test, $\alpha=0.05$

$D O=$ Detrusor overactivity; $D C B C=$ Decreased cystometry bladder capacity,

$D O+D C B C=$ Detrusor overactivity with decreased cystometry bladder capacity.

Table II: Treatment response to urotherapy among children with different urodynamic findings.

\begin{tabular}{l|cccc}
\hline Urodynamic & \multicolumn{4}{|c}{ Treatment result of one-month follow-up $(\mathrm{n})$} \\
\cline { 2 - 5 } study finding $(\mathrm{n})$ & $\mathrm{SR}(\mathrm{n})$ & PR $(\mathrm{n})$ & NR $(\mathrm{n})$ & P-value \\
\hline Normal $(135)$ & 93 & 36 & 6 & $<0.001 \#$ \\
DO (45) & 27 & 11 & 7 & \\
DCBC (30) & 10 & 13 & 7 & \\
DO+DCBC (22) & 3 & 7 & 12 & \\
\hline
\end{tabular}

Treatment response difference between different groups

\begin{tabular}{llr}
\hline Normal Vs & DO & $0.148^{*}$ \\
& DCBC & $<0.001^{*}$ \\
& DO+DCBC & $<0.001^{*}$ \\
\hline DO Vs & DCBC & $0.041^{*}$ \\
& DO+DCBC & $<0.001^{*}$ \\
\hline DCBC Vs & DO+DCBC & $0.019^{*}$
\end{tabular}

\#Kruskal-Wallis, $\alpha=0.05$. Treatment response difference among the four groups.

*Mann-Whitney test, $\alpha=0.05$.

7 years, the frequency of DCBC or DO+DCBC was significantly higher than children ranging from 3 to 7 years of age $(p=0.012)$.

All patients got follow-up at the end of one-month standard urotherapy and pelvic floor training after the UDS.

According to the evaluation criterion described earlier, of the 232 patients, $57.3 \%$ (133/232) children showed SR, $28.9 \%(67 / 232)$ children had PR, with the leftover $13.8 \%$ (32/232) children showed NR to the one-month urotherapy and pelvic floor training.

For the 86 children ranging from 3 to 5 years, $8.1 \%(7 / 86)$ showed NR to urotherapy, $29.1 \%(25 / 86)$ got PR, with the leftover $62.8 \%(54 / 86)$ showed SR. For patients from 5 to 7 years, $13.4 \%(11 / 82)$ were found with NR, $28.0 \%$ (23/82) with PR, 58.5\% (48/82) with SR. For the 64 patients older than 7 years, $21.9 \%$ (14/64) showed NR, $29.7 \%(19 / 64)$ showed PR, with the leftover $48.4 \%$ (31/64) showed SR. A Spearman's correlation was run to determine the relationship between the patient's age and treatment response to urotherapy. There was a weak negative monotonic correlation between age and treatment response to urotherapy (Spearman's correlation, $r s=-0.138, n=232, p=0.035$ ).

For the 135 patients with normal bladder function, $68.9 \%$ (93/135) children showed SR, 26.7\% (36/135) showed $\mathrm{PR}$, and $4.4 \%(6 / 135)$ showed NR to urotherapy. Of the 45 children with DO, $60.0 \%$ (27/45) achieved SR, $24.4 \%$ (11/45) had PR, and $15.6 \%$ (7/45) showed NR. For the
30 patients found with DCBC in the UDS, 33.3\% (10/30) attained SR, 43.3\% (13/30) had PR, and 23.3\% (7/30) showed NR. When evaluating the 22 children with DO+DCBC, 13.6\% (3/22) achieved SR, 31.8\% (7/22) had $P R$, and $54.5 \%$ (12/22) showed NR to urotherapy.

No statistically significant difference was observed between children with normal bladder function and children with DO $(p=0.148$, Mann-Whitney test $)$ to urotherapy. But for children with normal bladder function, the treatment response to urotherapy was significantly better than children with DCBC or DO+DCBC $(p<0.001$, Mann-Whitney test). Similar significant difference was also observed between children with $\mathrm{DO}$ and $\mathrm{DO}+\mathrm{DCBC}$ $(p<0.001$, Mann-Whitney test, Table II).

\section{DISCUSSION}

The definition of $O A B$ is based on symptoms only and allows establishing an initial treatment without having to go through other complex exam, but this may cause confusion in the diagnosis and evaluation of the problem.11,12 Especially, when the symptoms of $O A B$ showed resistant to the initial treatment, how should doctors choose other treatment strategies? If anticholinergic agents were routinely chosen as the second line therapy for $O A B$, it is inevitable that there will be more children faced with the problem of anticholinergic side effects.

Though mostly regarded as a self-limiting condition that resolves in most cases over time, the symptoms could last for months to years, which can disrupt daily activities and decrease the quality of life. ${ }^{6}$ Some studies indicated that if the condition continues over a long period of time, bladder wall thickening may occur, for which the capacity of bladder will decrease. 3 The decreased bladder capacity may further aggravate the urinary frequency, thus a vicious circle of overactive bladder was developed. In the present study, higher prevalence of DCBC or DO+DCBC was observed in children older than 7 years of age, and a weak negative correlation between age and treatment response to urotherapy was observed, which could further verify the viewpoint. Children's age was found to be a negative predictor of training result, which is not in line with other publications, 13 the reason for which is that in this study, $\mathrm{DCBC}$ was more frequently found in the older children.

The pathophysiology of $O A B$ has not been well understood. Altered function of the detrusor muscle, peripheral motor and sensory nerves, and central neural elements all contribute to the condition. ${ }^{14}$ The dysfunctional voiding of children is associated with the irregular and fluctuating muscle contractions of urethral sphincter or pelvic floor. 15 So, it is essential to understand the differential diagnosis of $O A B$, so that it can be treated accordingly. 
It is believed that UDS may help to assess the pathophysiology of $\mathrm{OAB}, 16$ but there were reports showing that DO and pelvic floor electromyography (EMG) could not predict the response to antimuscarinic treatment or urotherapy. 17 So, it is recommended that UDS should not be used in the initial evaluation of patients with $\mathrm{OAB}$, but UDS should be considered in the presence of refractory or complex symptoms.17,18 De Jong et al. stated that invasive urodynamic studies, such as cystometry, is reserved for patients for whom the outcome of such studies is expected to change the therapeutic regime. ${ }^{19}$ In the present study, UDS was carried out for the OAB symptoms which were resistant to three- to five-month standard urotherapy comprising education of the child and family, appropriate fluid intake, and regular optimal voiding. As urotherapy contains another category of specific interventions of urotherapy which include pelvic floor muscle awareness, biofeedback training and neuromodulation, during our UDS, pelvic floor muscle contraction was guided when urgency attacked. After UDS evaluation, all patients were asked to continue standard urotherapy and pelvic floor muscle training for another one month, then treatment response was evaluated. Overall, the present results show that patients with symptoms of OAB but with normal bladder function respond equally well to those with DO to urotherapy. The treatment response to standard urotherapy with pelvic floor training was suboptimal for children with DCBC, which was in line with the findings of another publication where a lower bladder volume observed in UDS may indicate a more severe clinical substrate and thus predict a poorer treatment response. 13 For these children, other treatment strategies, such as anticholinergic agents, should be considered. Moreover, the UDS could help to make treat-ment more individualised. When empiric treatment has failed, UDS is very important in diagnosing OAB and directing treatment. ${ }^{14}$

In this study, pelvic floor awareness was guided during UDS which may help patients to contract the pelvic floor muscle correctly, and there is study showing that bladder training and pelvic floor muscle training have significant treatment response. 8 This is somewhat like a biofeedback and is the possible mechanism why the treatment response was much better in the one-month urotherapy after the UDS.

So far, the correlation between DO and OAB has not yet been understood. Most studies tried to characterise OAB by whether or not DO was verified by UDS, but the relationship between $\mathrm{DO}$ and the pathophysiology of $\mathrm{OAB}$ was neglected. Some studies demonstrated that patients with DO may show a more severe form of OAB. ${ }^{14}$ What is more, UDS can provide other information of lower urinary tract, such as CBC, bladder compliance, bladder outlet obstruction and the state of sphincter. For children with $\mathrm{OAB}$, it is difficult to judge whether the decreased voiding volume is caused by DCBC or frequency, and during UDS, infusion was suspended when urgency attacked, which may help to reach the maximum $C B C$ close to reality. The treatment options for $\mathrm{OAB}$ are divided into three levels. The first-line treatment is behavioral therapy, which includes urotherapy, bladder training and pelvic floor muscle training. 20 The benefits of urotherapy are significant and patients just carry little to no risk of adverse effects. 7 The second-line treatment is medication. Antimuscarinics have remained the mainstay of treatment in children with overactive bladder when urotherapy and biofeedback could not get symptom remission, but the children will be faced with adverse effects such as dry mouth, constipation, blurred vision and cognitive adverse effects. ${ }^{3}$ When patients do not improve with first- and second-line treatment for $\mathrm{OAB}$, then the third-line treatment, which includes neuromodulation of the nerves controlling bladder function should be considered. ${ }^{7}$ The third-line treatment includes peripheral tibial nerve stimulation, sacral neuromodulation and botulinum toxin $A$ injection into the bladder, all of which have been approved by FDA.

In the present study, patients with symptoms of $O A B$, but without DCBC, respond better to those with DCBC to urotherapy. There are many factors such as DO, CBC, sphincter state, and social psychological factors that may influence the treatment effect of OAB. It may be argued that $D C B C$ is a possible negative influence factor for the treatment response of $\mathrm{OAB}$. The correlation between $O A B$ and $D C B C$ is still not understood. As there was no long-term follow-up, a further study is needed to investigate the pathophysiology of OAB. For children with DCBC and urotherapy-resistant OAB, other treatment strategies, such as antimuscarinic treatment, should be considered.

There are still some limitations to this study. As known, constipation is a very important aspect in children with urinary symptoms. 21 Though children with constipation were excluded from this study, but bowel movement data was not collected and analysed for children included, which may influence the choice of treatment, strategy and evaluation of treatment effect.

\section{CONCLUSION}

For OAB children, age may be one of the negative influence factors to the treatment effect of urotherapy, though the correlation is weak. Urodynamic verification of $\mathrm{DO}$ is not a prerequisite for urotherapy treatment response. Children with DCBC verified by UDS may suggest a poorer treatment response to urotherapy. If the OAB symptoms showed resistant to urotherapy, UDS is worth to be considered and may help to guide the correct pelvic floor muscle contraction and choose other treatment strategies. Further study on the correlation between DCBC and the pathophysiology of OAB is needed. 


\section{ETHICAL APPROVAL:}

The study has been approved by the Ethics Committee of Children's Hospital of Chongqing Medical University.

\section{PATIENTS' CONSENT:}

All patients signed informed consent for this study.

\section{CONFLICT OF INTEREST:}

Authors declared no conflict of interest.

\section{AUTHORS' CONTRIBUTION:}

CW: Made substantial contributions to the conception or design of the idea or the acquisition; analysed data; drafted the work; revised it critically for important intellectual content.

LK: Helped in writing the manuscript.

$\mathrm{XL}, \mathrm{TL}, \mathrm{DH}$ : Participated in the design and coordination of the paper.

JG: Participated in the design; final approval of the version to be published; agreement to be accountable for all aspects of the work.

\section{REFERENCES}

1. Stewart WF, Van Rooyen JB, Cundiff GW, Abrams P, Herzog AR, Corey $\mathrm{R}$, et al. Prevalence and burden of overactive bladder in the united states. World J Urol 2003; 20:327-36.

2. Bauer SB, Nijman RJ, Drzewiecki BA, Sillen U, Hoebeke P, International children's continence society standardization report on urodynamic studies of the lower urinary tract in children. Neurourol Urodyn 2015; 34:640-7.

3. Franco I. Pediatric overactive bladder syndrome. Pediatric Drugs 2007; 9:379-90.

4. Coyne KS, Sexton CC, Kopp ZS, Ebel-Bitoun C, Milsom I, Chapple $\mathrm{C}$. The impact of overactive bladder on mental health work productivity and health-related quality of life in the UK and Sweden results from epiluts. BJU int 2011; 108:1459-71.

5. Fitzgerald MP, Thom DH, Wassel-Fyr C, Subak L, Brubaker L, Van Den Eeden SK, et al. Childhood urinary symptoms predict adult overactive bladder symptoms. J Urol 2006; 175:989-93.

6. Sarica K, Yagci F, Erturhan S, Yurtseven C. Conservative management of overactive bladder in children evaluation of clinical and urodynamic results. J Pediatr Urol 2006; 2:34-9.

7. Willis-Gray MG, Dieter AA, Geller EJ. Evaluation and management of overactive bladder strategies for optimizing care. Res Rep Urol 2016; 8:113-22.
8. Rizvi RM, Chughtai NG, Kapadia N. Effects of bladder training and pelvic floor muscle training in female patients with overactive bladder syndrom: A randomized controlled trial. Urol Int 2018; 100:420-7.

9. Padilla-Fernandez B, Castro-Diaz DM. Combination therapy for overactive bladder: Should we define refractoriness? Eur Urol 2019; 76:780-1.

10. Ali M, Grogan S, Powell S, Staniford L, Nazir J, Landeira M, et al. Qualitative analysis of factors influencing patient persistence and adherence to prescribed overactive bladder medication in UK primary care. Adv Ther 2019; 36:3110-22.

11. Austin PF, Bauer SB, Bower W, Chase J, Franco I, Hoebeke P, et al. The standardization of terminology of lower urinary tract function in children and adolescents update report from the standardization committee of the international children's continence society. Neurourol Urodyn 2016; 35:471-81.

12. Truzzi JC, Gomes CM, Bezerra CA, Plata IM, Campos J, Garrido GL, et al. Overactive bladder 18 years part II. Int Braz J Urol 2016; 42:199-214.

13. Meijer EF, Nieuwhof-Leppink AJ, Dekker-Vasse E, de JoodeSmink GC, de Jong TP. Central inhibition of refractory overactive bladder complaints: Results of an inpatient training program. J Pediatr Urol 2015; 11:21e1-5.

14. Rutman MP, Cha DY, Blaivas JG. How do urodynamics findings influence the treatment of the typical patient with overactive bladder? Curr Urol Rep 2012; 13:370-8.

15. Austin PF, Bauer SB, Bower W, Chase J, Franco I, Hoebeke P, et al. The standardization of terminology of lower urinary tract function in children and adolescents. J Urol 2014; 191:1863$5 \mathrm{e} 13$.

16. Flisser AJ, Blaivas JG. Role of cystometry in evaluating patients with overactive bladder. Urology 2002; 60 (5 Suppl 1): 33-42.

17. Rovner ES, Goudelocke CM. Urodynamics in the evaluation of overactive bladder. Curr Urol Rep 2010; 11:343-7.

18. Al-Zahrani AA, Gajewski J. Urodynamic findings in women with refractory overactive bladder symptoms. Int J Urol 2016; 23:75-9.

19. de Jong TP, Klijn AJ. Urodynamic studies in pediatric urology. Nat Rev Urol 2009; 6:585-94.

20. Gormley EA, Lightner DJ, Faraday M, Vasavada SP. Diagnosis and treatment of overactive bladder (non-neurogenic) in adults AUA/SUFU guideline amendment. J Urol 2015; 193:1572-80.

21. Muhammad S, Nawaz G, Jamil I, Ur Rehman A, Hussain I, Akhter S. Constipation in pediatric patients with lower urinary tract symptoms. J Coll Physicians Surg Pak 2015; 25:815-8. 